\title{
Optimised uncertainty and cost operating characteristics: new tools for conformity assessment. Application to geometrical product control in automobile industry
}

\author{
L.R. Pendrill* \\ SP Technical Research Institute of Sweden, Measurement Technology Box 857, SE-501 15 Borås, Sweden
}

Received: 5 August 2010 / Accepted: 20 August 2010

\begin{abstract}
Translating measurement uncertainty into terms of effective impact associated with manufacture, testing and incorrect assessment gives a more "stakeholder" motivated (and ultimately optimised) approach to decision-making in conformance assessment. Recently developed decision-theory tools include the "optimized uncertainty" methodology and the "operating cost characteristic". Overall costs, E, consisting of a sum of testing costs, $D$, and the costs, $C$, associated with customer risk, can be calculated with the expression:

$$
E(\eta, \sigma)=D(\eta, \sigma)+C(\eta)=\frac{D}{\sigma^{2}}+\int_{\eta \notin R_{P V}} C(\eta) \cdot g(\eta \mid \hat{\eta}) \cdot d \eta
$$

with $\hat{\eta} \in R_{P V}$, where $R_{P V}$ denotes the region of permissible values and $\sigma$ is a measure of dispersion. A complete, 3D surface of overall cost can indicate the optimum level of measurement effort of these two ranges, as recently published by the author in a wide range of applications: optimized acceptance sampling; optimized testing of measurement instruments; and an analysis of optimised calibration intervals and "guard-banding". This approach is illustrated in the present work for the example of geometrical product control in the car industry, specifically the gap in vehicle closure panels taking account of customer dissatisfaction.
\end{abstract}

Keywords: Optimised; uncertainty; cost; GPS; automobile

\section{Introduction}

A well-known story [1] recounts how it was found that a main selling factor in favour of products of one car manufacturer over another was the ease with which car doors were opened and closed. A subsequent investigation narrowed this down as being due to a tighter tolerance on the geometrical specification of the door frame of the more popular car.

In the present paper, this case is presented as an example of an optimised uncertainty analysis [2-8]. Conformity assessment in the manufacture of vehicles will be examined in terms of geometrical product specifications $[9,10]$. Measurement uncertainties will be evaluated based on "real data" from a modern measurement instrument manufacturer for gap determination in vehicle closure panels. Thereafter, a discussion of costs - for measurement and for the consequences of incorrect decisions of conformity - will be given.

In any conformity assessment task, a balance has to be struck between expenditure on inspecting a product and the potential costs associated with various risks, to both

^ Correspondence: leslie.pendrill@sp.se the supplier and customer, associated with in-correct decisions arising from limited measurement accuracy and test uncertainties. Specifically, customer dissatisfaction will be modelled with a Taguchi cost model included for the first time in an optimised uncertainty analysis.

\section{Conformity, product and measurement quality}

The aim of conformity assessment of a type of entity is to assess conformance of actual values of a characteristic of, in general, a sample of typical entities with respect to specification limits on characteristic values, after compensating and allowing as far as possible for measurement quality variations [11]. Thus, in addition to assessment of actual product conformity, specifications are also set on the required measurement quality which has to be assessed for compliance as well.

\subsection{Product conformity assessment}

Specifications are set by the manufacturer on the gap in vehicle closure panels. Let us assume that a particular 


\section{Results Correlation \\ AutoFit and manual tools can correlate}

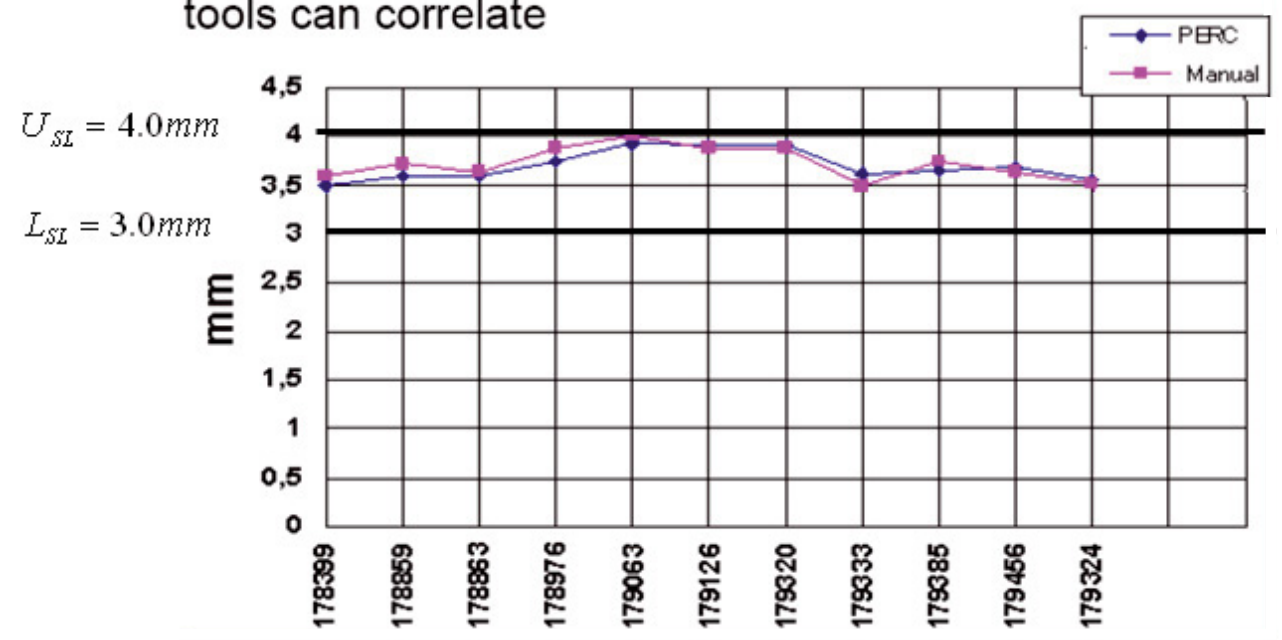

Fig. 1. Measurement of the gap in vehicle closure panels using two measurement methods ("PERC" and "Manual") [12].

gap should lie in the region of permissible values $3 \mathrm{~mm}$ to $4 \mathrm{~mm}$. Figure 1 shows some typical data from actual measurements.

Typical questions in this context, as exemplified by an instrument manufacturer are [12]: Are gap and flushness acceptable? Are problems unique to specific vehicles? Has unusual variation found its way into the assembly process? Where do the fitters make corrections? How much correction is required?

To answer questions such as these, one needs first to assess how much of the apparent product variation observed is caused by limited measurement quality. Thereafter, the observed variations have to be judged in terms of ultimate product quality and value, and whether it is worthwhile adjusting the manufacturing process and/or investing in more measurement capability.

\subsection{Measurement uncertainty}

There is initially little indication of measurement uncertainty in the original data shown in Figure 1. Without some declaration of measurement quality, it is difficult to judge how much of the observed variation is true product variation - which is of course the prime object of the actual product conformity assessment.

In analysing measurement uncertainty, each major element in the measurement system (including the object itself - i.e. the vehicle closure panel gap) needs to be examined for potential sources of unknown measurement error, as illustrated in Figure 2.

According to the specification sheet about the measurement instrument provided by the manufacturer, the instrument has a quoted "accuracy" of $0.05 \mathrm{~mm}$. A type-B standard measurement uncertainty is therefore estimated from this specification by associating a trapezoidal distri- bution with the specified accuracy, which means dividing this quoted value by a factor $\sqrt{ } 2$.

An idea of the size of the measurement uncertainty associated with each measurement method employed can be obtained from the data provided by the instrument manufacturer, especially in the comparison of measurements with the new instrument compared with more traditional "manual" measurement of vehicle closure panel gap.

A least-squares fit is made to obtain the average difference between the two methods along a series of measurements made at different points along the vehicle closure panel.

A type-A evaluation of measurement uncertainty can be based on the observed scatter of the two methods (Fig. 3). The calculated standard deviation s.d.(Autofit - Manual) of this difference can be directly taken as a type-A component of uncertainty $u_{A, \text { automan }}$.

$$
u_{A, \text { automan }}=\text { s.d. }(\text { Autofit }- \text { Manual })=0.097 \mathrm{~mm} .
$$

A type-B evaluation of measurement uncertainty can be based on the observed bias between the two methods (also shown in Fig. 3). Instead of correcting for the bias, one associates instead a rectangular (uniform) distribution with the observed mean bias Mean(Autofit - Manual) of this difference. Half of the observed mean bias is divided by the corresponding factor $\sqrt{ } 3$ in order to estimate the standard measurement uncertainty $u_{B, \text { automan }}$.

$$
\text { Mean }(\text { Autofit }- \text { Manual })=-0.046 \mathrm{~mm}
$$

$$
\begin{aligned}
& u_{B, \text { automan }}=\frac{\text { Mean }(\text { Autofit }- \text { Manual })}{2 \cdot \sqrt{3}} \\
&=\frac{0.046}{2 \cdot \sqrt{3}} \mathrm{~mm}=0.013 \mathrm{~mm}
\end{aligned}
$$




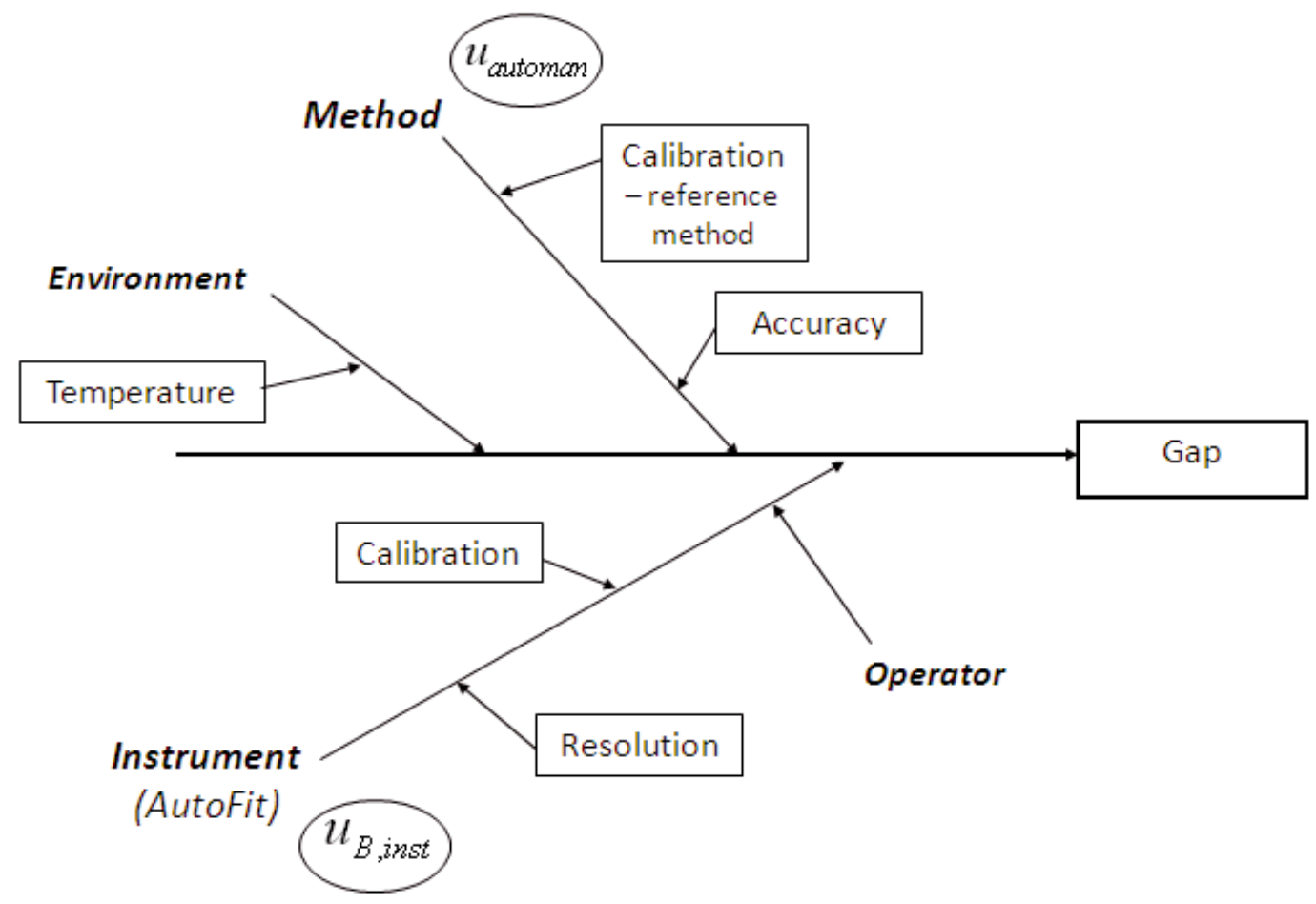

Fig. 2. Measurement uncertainty analysis as an Ishikawa diagram.

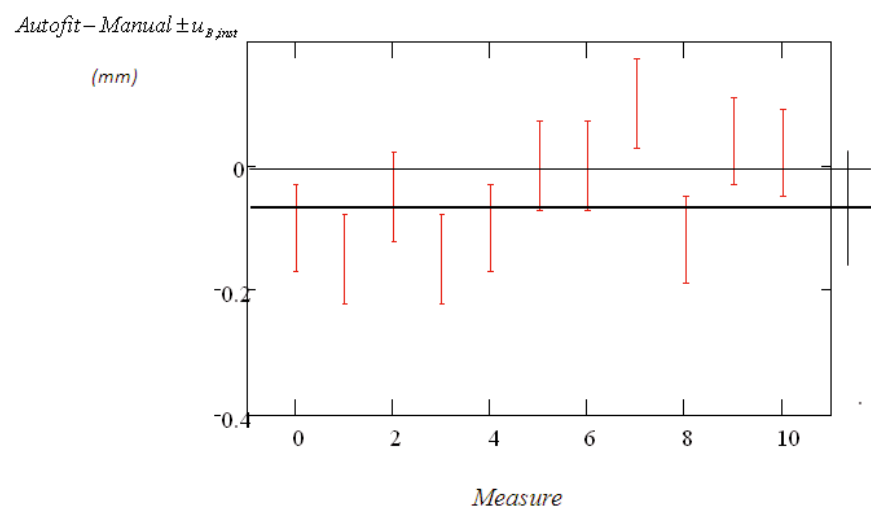

Fig. 3. Apparent differences between results of two measurement methods ("Autofit" and "Manual") for a series of gap measurements.

These two components of uncertainty in the measurement method are then combined (quadratically) to form the overall standard method uncertainty, $u_{\text {automan }}$.

$$
u_{\text {automan }}=\sqrt{\left(u_{A, \text { automan }}^{2}+u_{B, \text { automan }}^{2}\right)}=0.098 \mathrm{~mm} .
$$

This can be compared with the instrument uncertainty calculated before.

$$
u_{B, \text { inst }}=\frac{0.05 \mathrm{~mm}}{\sqrt{2}}=0.035 \mathrm{~mm} .
$$

\section{Decision-making in conformity assessment}

Although fully harmonised procedures for decision-making in the presence of measurement uncertainty are not available yet, three basic steps can be identified:

1. Set a limit on measurement uncertainty, usually as a specified maximum permissible uncertainty $(M P U)$ (or equivalently a minimum measurement capability $\left.C_{m, \min }\right)$ as some fraction of the maximum permissible product error $(M P E)$.

2. Decide on an acceptable location of the uncertainty interval with respect to the region of permissible (product) values.

3. Agree on acceptable levels of risk - both for the consumer and supplier - of in-correct decision of compliance, in percentage terms, but increasingly in terms of impact and costs.

Each of these three steps will now be illustrated in the example of conformity assessment of vehicle closure panel gap determination.

\subsection{Limits on measurement uncertainty}

The actual measurement uncertainty, $u_{\text {automan }}$, calculated in Section 2.2,

$$
\begin{aligned}
U_{\text {automan }}= & 2 \cdot u_{\text {automan }} \\
& =\sqrt{\left(u_{\text {A,automan }}^{2}+u_{B, \text { automan }}^{2}\right)}=0.2 \mathrm{~mm}
\end{aligned}
$$


seems to be somewhat high compared with the agreed maximum permissible (target) uncertainty.

$$
\begin{aligned}
C_{m, \min }= & \frac{U_{S L}-L_{S L}}{2 \cdot U_{\text {measure }}}>3 \quad M P U=\frac{M P E}{3} \\
& =\frac{U_{S L}-L_{S L}}{2 \cdot C_{m, \min }}=\frac{4.0-3.0}{2 \cdot 3} \mathrm{~mm}=0.17 \mathrm{~mm} .
\end{aligned}
$$

Most of the uncertainty appears to be associated with the scatter in measurement results with the two measurement methods. This would suggest that one should choose the "better" measurement method for conformity assessment purposes.

\subsection{Location of uncertainty interval}

We now analyse in more depth a particular test result, shown in Figure 1, namely observation 179063 , which lies close to the upper specification limit $U_{S L}$ of $4 \mathrm{~mm}$ gap.

Guard-banding is one technique to protect against incorrect conformity decisions caused by measurement uncertainty or entity dispersion, where the region of permissible values of the entity's quality characteristic is reduced in proportion to the actual measurement uncertainty or dispersion.

A "guard-band" is placed around each specification limit with a width equal to some fraction of the actual measurement uncertainty.

According to the standardised ISO Geometrical Product Specification (GPS) approach:

"Uncertainty of measurement always counts against the party who provides the proof of conformance or nonconformance and therefore makes the measurement" (Sect. 6.1 of ISO 14253-1:1998) [10].

Thus, in the case where the Supplier proves conformance with his estimated measurement uncertainty, then consumer risk (approving non-conforming product) is minimised by setting a guard-band on the upper specification limit:

$$
y_{m} \leqslant U_{S L}-h \cdot \sigma .
$$

In the case where the Consumer proves non-conformance with his estimated measurement uncertainty, then producer risk (failing conforming product) is minimised by setting a guard-band on the upper specification limit:

$$
y_{m} \geqslant U_{S L}+h \cdot \sigma .
$$

An acceptable guard-band factor, $h$, needs to be agreed upon in each case, but for the initial sake of argument, $h=$ 0.5 would imply that the test result, observation 179063 , at $y_{m}=3.90 \pm 0.18 \mathrm{~mm}$, needs to be compared with:

$$
U_{S L}-h \cdot u=4.00-0.5 \cdot u=3.96 \mathrm{~mm} .
$$

That is, acceptable when the Supplier proves conformance.

$$
U_{S L}+h \cdot u=4.00+0.5 \cdot u=4.04 \mathrm{~mm}
$$

i.e. there is no case for claiming non-conformance by the Consumer, according to the GPS rules.

\subsection{Assessment of risks}

The above rules of decision-making appear to be largely arbitrary. A first step to deciding which test results are acceptable to the end-user is to assess the percentage risk of incorrect compliancy decisions.

The measured gap in the vehicle closure panels lies within, but close to - for instance, at observation 179063 , the upper specification limit on the gap.

The associated consumer risk - accepting incorrectly a non-conforming product - is calculated to be $11.5 \%$ using the following expression:

$$
\begin{aligned}
p_{c} & =\operatorname{Pr}\left(Y \geqslant U_{S L, x} \mid y_{m}, u\right)=\int_{U_{S L, x}}^{\infty} g_{m}\left(y \mid y_{m}, u\right) \cdot d y \\
& =\int_{U_{S L, x}}^{\infty} \frac{1}{\sqrt{2 \pi} \cdot u} \exp \left[-\frac{\left(y-y_{m}\right)^{2}}{2 \cdot u^{2}}\right] \cdot d y=0.115
\end{aligned}
$$

which measures the percentage consumer risk as the integrated area (cumulative) under the Gaussian distribution (assumed to be associated with the measurement uncertainty probability distribution function) which lies above the upper specification limit.

Obviously, the level of consumer risk will vary as the test result is swept over the specification limit. An operating characteristic curve for specific conformity assessment by variable is obtained by evaluating the cumulative (Normal) probability of conformity, given by expression (1) over a range of quantity values of $L_{S L}-h \cdot u_{m} \leqslant$ $y_{m} \leqslant L_{S L}+h \cdot u_{m}$ for a given test uncertainty, $u_{m}$, when measuring one item and "guard-band" factor $h$.

Customer and supplier can use such operating characteristic curves [13] to agree on:

- a maximum level, $\beta$, of consumer risk - say, $10 \%$ where the uncertainty interval is located at the value $L Q L$ (limiting quality level) of the quality characteristic. Characteristic entity values further away from (below) the (upper) specification limit, will have probabilities of in-correctly accepting a non-conforming entity less than $\beta$.

- a minimum level, $\alpha$, of supplier risk - say, 95\% where the uncertainty interval is located at the value $A Q L$ (acceptable quality level) of the quality characteristic. Characteristic entity values further away from (above) the (upper) specification limit, will have probabilities of correctly rejecting a non-conforming entity greater than $\alpha$.

Once again, if a certain level of percentage risk is acceptable or not for the parties involved in the conformity assessment - in the present case of vehicle closure panel gap - can still be debated.

The final step in deciding conformity is to estimate for either Supplier or Consumer - what levels of risks are of incorrect decisions in terms of impact, often cost. 


\section{Assessment of impact. Optimised uncertainty}

Of use to the decision-maker in conformity assessment are not only estimates of the probability of quantity values being within certain intervals but also the costs associated with product quantity errors. These costs, which are arguably of prime interest to the professional decisionmaker, are typically associated with:

- actual product errors;

- apparent quantity errors and the risks of incorrect decision-making associated with measurement uncertainty;

- and the cost associated with obtaining a certain level of measurement uncertainty in conformance testing.

These incorrect decisions in turn lead to a variety of consequences, with associated risks and costs, such as unnecessary re-manufacturing by the supplier as well as various consequences for the customer, arising from incorrect measures of quantity, poor product performance, not to mention possible litigation where disputes in conformity assessment end up in a legal process in court. Various cost models and how economics is introduced in risk assessment in conformity assessment are dealt with in this final section for the particular case at hand, namely conformity assessment of vehicle closure panel gap.

Overall costs, E, balance:

(i) Test costs, $D$, here modelled as inversely proportional to the squared dispersion. $D=500 €$ Test cost @ $C_{m}=6$.

(ii) In general, the impact of a wrong decision in conformity assessment is expressed as a risk $\boldsymbol{R}$, defined as the probability $\boldsymbol{p}$ of the wrong decision occurring multiplied by the cost $C$ of the consequences of the incorrect decision: $\boldsymbol{R}=\boldsymbol{p} \times \boldsymbol{C}$ - a more general, historical expression of statistical expectation. Incorrect acceptance of a non-conforming object on inspection will lead to customer costs associated with out-of-tolerance product. Consequence costs, $C$, are here modelled as equal to the loss of sales per vehicle if the product is incorrectly passed. The customer would be sufficiently dissatisfied with the large gap so that he wants his money back!

$$
\begin{aligned}
& E\left(y_{m}, u\right)=D\left(y_{m}, u\right)+C\left(y_{m}\right) \\
&=\frac{D}{u^{2}}+\int_{\eta \notin R_{P V}} C(\eta) \cdot g\left(\eta \mid y_{m}\right) \cdot d \eta
\end{aligned}
$$

where $y_{m} \in R_{P V}$.

A common model which aims to account for the fact that the more a product deviates from nominal, the less satisfied will be the customer, is due to Taguchi [14] as expressed by his loss function:

$$
L_{\text {Taguchi }}=\frac{C_{n p}}{\left(\frac{U S L-L S L}{2}\right)^{2}} x^{2}
$$

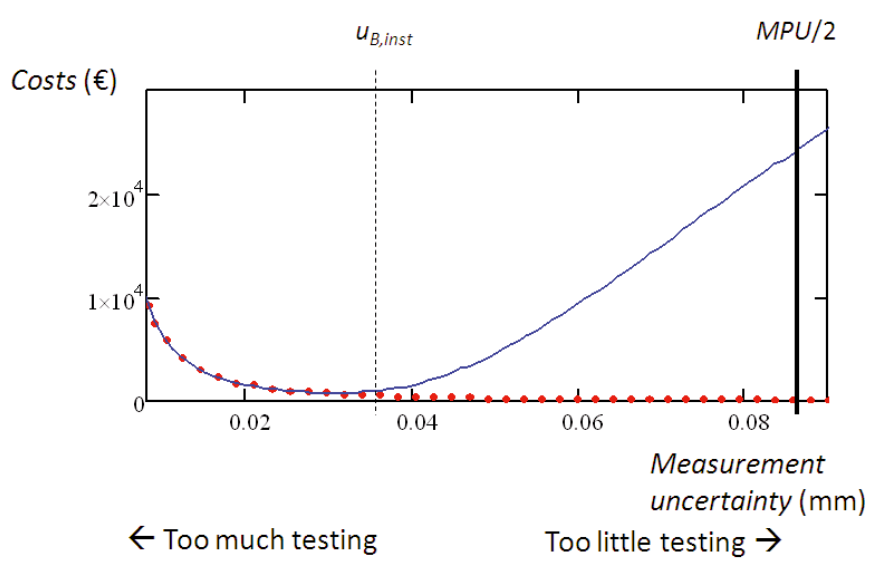

Fig. 4. Optimised uncertainty analysis for conformity assessment of vehicle closure panel gap for observation 179063 .

where the consumer risk losses are zero when entity error, $x$, is zero, and equal to $C_{n p}$ when entity error, $x$, is at either of the specification limits, USL or $L S L$.

In the present work, we point out that proper account of these losses is made by a "folding" of the squared-error term of the Taguchi loss function with the measurement uncertainty distribution, leading to the following original expression for consumer-related costs arising from customer dissatisfaction:

$$
\frac{P_{\text {customer }} C_{n p}}{\left(\frac{U S L-L S L}{2}\right)^{2}}\left[\int_{U S L_{x}}^{\infty} x^{2} \frac{1}{\sqrt{2 \pi} \cdot u_{\text {measure }}} e^{-\frac{\left(x-x_{\text {measure }}\right)^{2}}{2 \cdot u_{\text {measure }}^{2}}} d x\right]
$$

where typically $P_{\text {customer }}=0.01$, i.e. $10 \%$ is the fraction of customers who would reject the product if dissatisfied, with a consequence cost, $C_{n p}=30000 €$, for a product incorrectly passed which the customer subsequently rejects.

An optimised uncertainty analysis (Fig. 4), resulting from an evaluation of equation (2) over a range of (standard) uncertainties, indicates the minimum overall costs to be obtainable at an "optimum" measurement uncertainty of about one half of the originally agreed maximum permissible (target) uncertainty.

\section{Conclusions}

A complete, 3D surface of overall cost can indicate the optimum level of measurement effort over a range of test results (operating characteristic) and a range of uncertainties (optimum uncertainty) [3]. Such an analysis goes beyond the somewhat arbitrary "rules of thumb" often used to set limits on measurement uncertainty as some fraction of the product specification interval. This approach is illustrated here for the example of geometrical product control for vehicle closure panel gap determination in the car industry. After an analysis of typical data and associated measurement uncertainties, risks of incorrect decisions of conformity have been treated both according to present-day GPS standards in percentage risk terms as 
well as an optimised uncertainty model made specifically with cost models taking account of measurement effort and customer dissatisfaction.

Acknowledgements. The author has benefited from participation in the Joint Committee for Guides in Metrology (JCGM), Working Group 1. This work has been partially financed by grant National Metrology of VINNOVA, the Swedish Innovation Agency.

\section{References}

1. D. Swyt, Metrological Issues in Precision-Tolerance Manufacturing: A Report of a NIST Industry-Needs Workshop, J. Res. NIST 98, 245-252 (1993)

2. T. Fearn, S. Fisher, M. Thompson, S. Ellison, A decisiontheory approach to fitness for purpose in analytical measurement, Analyst 127, 818-824 (2002)

3. L.R. Pendrill, Operating "cost" characteristics in sampling by variable and attribute, Accred. Qual. Assur., http://dx.doi.org/10.1007/s00769-008-0438-y (2008)

4. L.R. Pendrill, H. Källgren, Optimised measurement uncertainty and decision-making in the metering of energy, fuel and exhaust gases, Izmerite'lnaya Technika (Measurement Techniques) 51, 370-377 (2008), http://dx.doi.org/10.1007/s11018-008-9047-8

5. L.R. Pendrill, Optimised measurement uncertainty and decision-making in conformity assessment, NCSLi Measure 2, 76-86 (2007)

6. L.R. Pendrill, Optimised measurement uncertainty and decision-making when sampling by variables or by attribute, Measurement 39, 829-840 (2006), Advanced Mathematical Tools for Measurement in Metrology and Testing, http://dx.doi.org/10.1016/j.measurement. 2006.04.014

7. L.R. Pendrill, H. Källgren, Decision-making with uncertainty in attribute sampling, Advanced Mathematical and Computational Tools in Metrology VII, in Series on Advances in Mathematics for Applied Sciences (World Scientific, Singapore, 2006), Vol. 72, pp. 212-220

8. L.R. Pendrill and $H$ Källgren, Exhaust gas analysers and optimised sampling, uncertainties and costs, Accred. Qual. Assur. 11, 496-505 (2006), http://dx.doi.org/10.1007/s00769-006-0163-3

9. http://metrology.wordpress.com/ measurement-process-index/ geometrical-product-specifications-gps/

10. ISO 14253-1:1998, Geometrical Product Specification (GPS) - Inspection by measurement of workpieces and measuring instruments - Part 1: Decision rules for proving conformance or non-conformance with specifications.

11. JCGM WG1, Evaluation of measurement data - The role of measurement uncertainty in deciding conformance to specified requirements, draft GUM Supplement JCGM 106 March 2010, Joint Committee for Guides in Metrology, http://www.bipm.org/en/committees/jc/jcgm/

12. http://www.perceptron.com/downloads/AutoFitprofile. pdf

13. D.C. Montgomery, Introduction to Statistical Quality Control (John Wiley \& Sons, Hoboken, NJ, 1996)

14. G. Taguchi, Taguchi on Robust Technology (ASME Press, New York, 1993) 